\title{
CXCL5 Downregulation in Villous Tissue Is Correlated With Recurrent Spontaneous Abortion
}

OPEN ACCESS

Edited by:

Sha Wu,

Southern Medical University, China

Reviewed by:

Maikel Peppelenbosch,

Erasmus Medical Center, Netherlands

Ling Niu,

lowa State University, United States

*Correspondence:

Tailang Yin

reproductive@whu.edu.cn

Yan Zhang

peneyyan@mail.ustc.edu.cn

${ }^{t}$ These authors have contributed equally to this work and share first authorship

Specialty section:

This article was submitted to Immunological Tolerance and Regulation,

a section of the journal

Frontiers in Immunology

Received: 31 May 2021 Accepted: 30 August 2021 Published: 17 September 2021

Citation:

Zhang S, Ding J, Wang J, Yin T, Zhang Y and Yang J (2021) CXCL5

Downregulation in Villous Tissue Is

Correlated With Recurrent

Spontaneous Abortion.

Front. Immunol. 12:717483.

doi: 10.3389/fimmu.2021.717483

\section{Sainan Zhang ${ }^{1 \dagger}$, Jinli Ding ${ }^{1 \dagger}$, Jiayu Wang ${ }^{1 \dagger}$, Tailang Yin ${ }^{1 *}$, Yan Zhang ${ }^{2 *}$ and Jing Yang ${ }^{1}$}

${ }^{1}$ Reproductive Medical Center, Renmin Hospital of Wuhan University \& Hubei Clinic Research Center for Assisted Reproductive Technology and Embryonic Development, Wuhan, China, ${ }^{2}$ Department of Clinical Laboratory, Renmin Hospital of Wuhan University, Wuhan, China

Recurrent spontaneous abortion (RSA) affects $5 \%$ of childbearing-age women worldwide. Inadequate trophoblast invasion is one of the main reasons for the development of RSA; however, the underlying molecular mechanisms for RSA have not been fully understood, and further explanation is urgently needed. C-X-C motif chemokine ligand 5 (CXCL5) is reported to contribute to the invasion of cancer cells, and its aberrant expression is associated with the cellular process of tumor pathology. Considering the high behavioral similarity between trophoblast cells and cancer cells, we hypothesized that CXCL5 may influence trophoblast invasion, and its expression levels in villous tissue may be correlated with RSA. In this study, we firstly investigated the CXCL5 expression in placental villous tissues of 15 RSA patients and 13 control patients, and the results showed that CXCL5 levels were significantly lower in villous tissue from RSA patients than those of the controls. Further in vitro experiments presented that recombinant human CXCL5 can enhance trophoblast migration, invasion, and epithelial-to-mesenchymal transition (EMT) process. We also demonstrated that CXCL5 exerted these effects on trophoblast cells through PI3K/AKT/ERK1/2 signaling pathway. In conclusion, these data indicate that CXCL5 downregulation in human villous tissue is correlated with RSA. Additionally, we found that estrogen, progesterone, human chorionic gonadotropin, and decidual stromal cells can regulate CXCL5 and chemokine receptor 2 (CXCR2) expression of trophoblast in a cell manner.

Keywords: CXCL5, recurrent spontaneous abortion, invasion, migration, trophoblast

Abbreviations: RSA, recurrent spontaneous abortion; EVTs, extravillous trophoblasts; EMT, epithelial-to-mesenchymal transition; ENA-78, epithelial neutrophil-activating peptide 78; CXCL5, C-X-C motif chemokine ligand 5; CXCR2, chemokine receptor 2; DSCs, decidual stromal cells; E, estrogen; P, progesterone; HCG, human chorionic gonadotropin; PBS, phosphatebuffered saline; RT-PCR, quantitative real-time PCR; IHC, immunohistochemistry; FBS, fetal bovine serum. 


\section{INTRODUCTION}

Recurrent spontaneous abortion (RSA) is defined as two or more times of consecutive miscarriages before 20 weeks of gestation and impacts approximately 5\% of childbearing-age women throughout the world (1-3). There is no unified theory in the pathogenesis of RSA. Several known factors for RSA include endocrine diseases, genetic abnormalities, immune diseases, and anatomical abnormalities, and approximately 50\% of RSA cases are unexplained (4). The underlying molecular and cytological mechanisms for RSA also remain largely enigmatic. As the most common pregnancy-associated complication, RSA seriously disturbs the physical and mental health of the female population and is also frustrating for the physician. Trophoblast invasion is a key event during pregnancy and plays a vital role in the process of embryo implantation and placentation (5). In the early stage of pregnancy, trophoblast cells from the trophectoderm differentiate into two main lineages, villous cytotrophoblasts (VCTs) and extravillous trophoblasts (EVTs). Subsequently, EVTs undergo migration and invasion into the maternal decidua and myometrium, during which process embryos are anchored to the uterine wall and the uterine vessels are remodeled to form the low-resistance spiral arteries $(6,7)$. Proper trophoblast invasion is necessary for a successful pregnancy. Studies show that poor trophoblast invasion is related to a series of pregnancy complications, including RSA, preeclampsia, and fetal growth restriction (8, 9). Inadequate invasion of the trophoblast is even reported to be one of the main reasons for RSA (10-12). Therefore, exploring the factors that affect trophoblast invasion is of great significance for improving our understanding of the pathogenesis of RSA. Many molecules including hormones, chemokines, and growth factors, are associated with trophoblast invasion within the maternal-fetal microenvironment. Of these, chemokines are a large family of small-molecular-weight peptides that are initially involved in the pro-inflammatory process (13). Recently, studies have demonstrated that a wide range of chemokines are expressed at the maternal-fetal interface, and these chemokines can directly participate in the regulation of trophoblast invasion and the establishment of maternal-fetal tolerance $(14,15)$.

$\mathrm{C}-\mathrm{X}-\mathrm{C}$ motif chemokine ligand 5 (CXCL5), also known as epithelial neutrophil-activating peptide 78 (ENA-78), is a member of the CXC chemokine subfamily and is originally identified in neutrophils (16). It has been demonstrated that CXCL5 is a potent mediator of neoangiogenesis and is mainly expressed in epithelial cancer cells and immune cells (17). Chorionic trophoblast and amniotic epithelium membranes have also been confirmed to express CXCL5 (18). Abnormal expression of CXCL5 is found to be correlated with a large number of diseases, such as autoinflammation, cancer, obesity, and diabetes (19-21). CXCL5 exerts its action by binding to its G-protein-coupled receptors chemokine receptor 2 (CXCR2), and the CXCL5/CXCR2 axis has been widely investigated in various types of cancers. Numerous studies have confirmed that CXCL5 can induce the epithelial-to-mesenchymal transition (EMT) process in cancer cells and thus promotes cancer cells invasion and metastasis $(22,23)$. Trophoblast cells and cancer cells share striking behavioral similarities in invasion, migration, and proliferation capacities (24). Whether CXCL5 can affect trophoblast invasion has not been reported. A previous study identified that human villi expressed CXCR2 (25). It was also found that as one of the ligands of CXCR2, IL-8, was expressed in human decidua and trophoblast and promoted trophoblast migration and invasion in an autocrine or paracrine manner (26). Similar results were found in CXCL3 (27). These findings draw our attention to the CXCL5. Additionally, one study evaluated the relationship between the levels of chemokine and the risk of miscarriage. The study found that elevated CXCL5 levels from serum samples were associated with an increased risk of miscarriage as the collection-outcome interval increased, although the authors did not observe statistical significance (28). Based on these results, we hypothesized that CXCL5 may exert an effect on trophoblast migration and invasion and thus could participate in the occurrence of RSA.

In the present study, we compared CXCL5 levels in the placental villous tissue of RSA patients and control patients. We also investigated its effects on the trophoblast and explored the underlying mechanism. Our results showed that the expression of CXCL5 was significantly lower in RSA patients. We also further demonstrated that CXCL5 can induce the EMT process through PI3K/AKT/ERK1/2 signaling pathway and thus promoted trophoblast invasion and migration. Collectively, these data provide the first evidence that CXCL5 downregulation in villous tissue is correlated with RSA. In addition, we found that local factors, including estrogen $(\mathrm{E})$, progesterone $(\mathrm{P})$, human chorionic gonadotropin (HCG), and decidual stromal cells (DSCs) regulated CXCL5 and CXCR2 expression of trophoblast cells.

\section{MATERIALS AND METHODS}

\section{Patients and Clinical Samples}

This study was approved by the ethics committee of Renmin Hospital of Wuhan University, and consent was obtained from each patient before sample collection. Fifteen patients with RSA and 13 control patients from the reproductive medical center in Renmin Hospital of Wuhan University were included between December 2017 and October 2019. Clinical data and placental villous tissue samples were obtained from the two groups. RSA was defined as the loss of two or more sequential pregnancies with the same partner before a gestational of 20 weeks. The exclusion criteria were as follows: a) symptoms of endocrine or metabolic diseases, such as hyperthyroidism and diabetes; b) karyotype abnormality; c) infection based on routine leucorrhea examination; and d) uterine abnormality. Women who had healthy pregnancies and underwent selective pregnancy terminations for non-medical reasons constituted the control group. The villous tissues were collected immediately following surgery: one portion was fixed with $4 \%$ paraformaldehyde for paraffin embedding in blocks, and the others portion was stored in liquid nitrogen. 


\section{Immunohistochemistry}

The paraffin-embedded villous tissues were cut into $4-\mu \mathrm{m}$-thick sections and dehydrated in a graded series of ethanol. Endogenous peroxidase activity was blocked with $3 \% \mathrm{H}_{2} \mathrm{O}_{2}$, and non-specific binding was blocked with $5 \%$ bovine serum albumin (BSA) for $15 \mathrm{~min}$. Next, the samples were incubated at $37^{\circ} \mathrm{C}$ with primary rabbit anti-human CXCL5 antibody (1:500; Affinity, Cat: DF9919), anti-E-cadherin (1:500; ProteinTech; Cat: 20874-1-AP) and anti-N-cadherin (1:100; ProteinTech; Cat: 22018-1-AP) antibodies. All sections were washed three times with PBS and then incubated with secondary antibodies. The reaction was detected with $3,3^{\prime}$-diaminobenzidine (DAB), and the sections were counterstained with hematoxylin. Five visual fields were selected, and the staining was observed under an Olympus BX51+DP70 microscope at $\times 200$ and $\times 400$ magnification. The images were analyzed with ImageJ (1.52a, National Institutes of Health, USA).

\section{Immunofluorescence}

The paraffin-embedded human villous tissues were cut into 2$\mu \mathrm{m}$-thick sections. Deparaffinization, hydration, and antigen retrieval of the sections were carried out under proper conditions. The samples were incubated with rabbit antihuman cytokeratin 7 (CK7) (1:100; ProteinTech, Cat: 15539-1AP) and rabbit anti-human CXCR2 (1:50; ProteinTech, Cat: 20634-1-AP) primary antibodies. After that, the samples were incubated with fluorescence-labeled secondary antibody for $1 \mathrm{~h}$ and counterstained with 4'-6-diamidino-2-phenylindole (DAPI) (Beyotime, Shanghai, China). A confocal laser scanning microscope (Olympus FV1000, Japan) was used to observe the fluorescence signal. Five visual fields with tissue were selected for analysis. The pixel intensity per unit area was assessed using ImageJ (1.52a, National Institutes of Health, USA).

\section{Cell Culture, Reagents, and Treatments}

HTR-8/SVeo cell line was obtained from the China Center for Type Culture Collection (Wuhan, China) and cultured in DMEM-F12 medium (Gibco, USA) supplemented with $10 \%$ fetal bovine serum (FBS) (Gibco, USA). Human endometrial stromal cells were purchased from the BeNa culture collection and induced toward DSCs according to a previous method, with some modifications (29, $30)$. HTR- 8 cells were seeded on a 6 -well plate $\left(2 \times 10^{5}\right.$ cells/well $)$ and placed in an incubator with $5 \% \mathrm{CO}_{2}$ at $37^{\circ} \mathrm{C}$. A co-culture model of HTR- 8 cells and DSCs was established via a Transwell co-culture system $(0.4-\mu \mathrm{m}$ pore size, Corning, USA). In brief, HTR-8 cells were seeded into the lower chambers, and DSCs were placed into the upper chambers at different ratios (DSCs: HTR-8 cells $1: 4 ; 1: 1 ; 2: 1$ ) for 48 h before harvest.

Recombinant human CXCL5 (Absin, Shanghai, China) was used at the concentration of 50 and $100 \mathrm{ng} / \mathrm{ml}$ according to the manufacturer's instruction. LY294002 (PI3K/AKT inhibitors) and PD98059 (ERK1/2 inhibitors) were purchased from MedChemExpress, China, and used at concentrations of 20 and $30 \mu \mathrm{M}$, respectively. Hormone concentrations used in the current experiment were E $\left(10^{-7} \mathrm{M}\right), \mathrm{P}\left(10^{-8} \mathrm{M}\right)$, and HCG (5 kU/L).

\section{Quantitative Real-Time PCR}

Total RNA was extracted from cells and tissues using TRIzol reagent (Invitrogen, USA) according to the manufacturer's instructions. Reverse transcription was conducted with the PrimeScript RT reagent kit (Takara, Japan). RT-PCR was performed with a SYBR Premix Ex Taq II kit (Takara, Japan) on a 7500 detection system (Applied Biosystems, Foster City, CA, USA). $2^{-\Delta \Delta} \mathrm{Ct}$ method was determined to calculate and quantify the gene expression. Primers were designed with computer assistance based on gene sequences available in GenBank, and the sequences of primers are listed in Table 1.

\section{Western Blotting}

Cells were harvested and lysed with radioimmunoprecipitation assay (RIPA) lysis buffer, and the lysates were centrifuged at $4^{\circ} \mathrm{C}$ for $15 \mathrm{~min}$ to collect the supernatant. A bicinchoninic acid (BCA) assay kit (Beyotime, Shanghai, China) was used to measure protein concentrations. After boiling with a $5 \times$ loading buffer (Beyotime, Shanghai, China) at $95^{\circ} \mathrm{C}$ for $5 \mathrm{~min}$, $40 \mu \mathrm{g}$ of protein of each sample was electrophoresed via $10 \%$ sodium dodecyl sulfate-polyacrylamide gel and transferred to polyvinylidene difluoride (PVDF) membranes (Millipore) for blocking $1 \mathrm{~h}$ at room temperature with 5\% BSA. The following primary antibodies were incubated together with the membranes overnight at $4^{\circ} \mathrm{C}$ : rabbit anti-E-cadherin (dilution 1:1,000; ProteinTech; Cat: 20874-1-AP), anti-N-cadherin (dilution 1:1,000; ProteinTech; Cat: 22018-1-AP), anti-vimentin (dilution 1:1,000; ProteinTech; Cat: 10366-1-AP), anti-GAPDH (dilution 1:1,000; ProteinTech; Cat: 10494-1-AP), and antitubulin (dilution 1:1,000; ProteinTech; Cat: 10094-1-AP). The secondary antibodies were incubated for $1 \mathrm{~h}$ at room temperature the next day. Finally, the protein bands were visualized with an enhanced chemiluminescence (ECL) detection system (Bio-Rad, Hercules, CA, USA), and the relative band intensities were calculated with ImageJ (1.52a, National Institutes of Health, USA).

\section{Invasion Assay}

A 24-well plate Transwell insert (8- $\mu$ m pore size, Corning, USA) coated with Matrigel matrix (Corning, USA) was used to detect the invasion ability of the cell. In brief, HTR- 8 cells $\left(2 \times 10^{4}\right)$ were seeded in the upper chamber of each insert in a $200 \mu \mathrm{l} \mathrm{FBS-free}$ DMEM-F12 medium. The lower chamber was filled with $500 \mu \mathrm{l}$ of DMEM-F12 medium containing 10\% FBS. The plate was

TABLE 1 | Primer sequences

\begin{tabular}{lll}
\hline Primers & & \multicolumn{1}{c}{ Sequences $\mathbf{( \mathbf { 5 } ^ { \prime } \mathbf { - 3 } \mathbf { ) }}$} \\
\hline GAPDH & $F$ & CACTGGGCTACACTGAGCAC \\
& $\mathrm{R}$ & AGTGGTCGTTGAGGGCAAT \\
CXCL5 & $\mathrm{F}$ & AGCTGCGTTGCGTTGTTAC \\
& $\mathrm{R}$ & TGGCGAACACTTGCAGATTAC \\
CXCR2 & $\mathrm{F}$ & CCTGTCTTACTITCCGAAGGAC \\
& $\mathrm{R}$ & TTGCTGTATTGTTGCCCATGT
\end{tabular}

GAPDH, glyceraldehyde 3-phosphate dehydrogenase; CXCL5, C-X-C motif chemokine ligand 5; CXCR2, chemokine receptor 2. 
placed in an incubator with $5 \% \mathrm{CO}_{2}$ at $37^{\circ} \mathrm{C}$ for $48 \mathrm{~h}$. Afterward, cells that had invaded the lower chamber were fixed with $4 \%$ paraformaldehyde, stained with $0.5 \%$ crystal violet, and quantified. The average cells number from five fields at a magnification of $\times 200$ was reported.

\section{Scratch Wound Healing Assay}

Cell migration ability was evaluated with scratch wound healing assay. When cells reached $80 \%-90 \%$ confluence, a scratch wound was made on the monolayer of cells with a $200-\mu l$ pipette tip and gently washed three times with PBS before the serum-free medium was added. The 6 -well plate was incubated with 5\% $\mathrm{CO}_{2}$ at $37^{\circ} \mathrm{C}$ for $48 \mathrm{~h}$. Pictures of the wound were taken at 0 and $48 \mathrm{~h}$. The wound area was calculated using ImageJ.

\section{Statistical Analysis}

Quantitative data were expressed as the mean \pm standard deviation (SD) and analyzed by independent t-test. Categorical data were compared by the Mann-Whitney U-test. All experiments were independently repeated at least three times. Figures were performed by GraphPad Prism version 6.0 (GraphPad Software, San Diego, CA). All $p$-values were twosided and statistical significance was established as $p<0.05$. All analyses were conducted using SPSS 22.0 (IBM SPSS, USA).

\section{RESULTS}

\section{Clinical Baseline Characteristics}

Collected data at baselines included maternal age, gestational week, body mass index, number of pregnancies, number of live births, and number of miscarriages. Detailed information is listed in Table 2. No differences were found between the RSA group and the control group in terms of age, gestational week, body mass index, and the number of pregnancies. In addition, the RSA group showed a higher number of miscarriages and a
TABLE 2 | Clinical characteristics of the population.

\begin{tabular}{|c|c|c|}
\hline Primers & Control $(n=13)$ & $\operatorname{RSA}(n=15)$ \\
\hline Age (years) & $30.17 \pm 2.05$ & $30.63 \pm 3.97$ \\
\hline $\mathrm{BMl}\left(\mathrm{kg} / \mathrm{m}^{2}\right)$ & $21.17 \pm 4.15$ & $22.74 \pm 3.89$ \\
\hline Gestation week & $7.17 \pm 2.15$ & $7.67 \pm 2.01$ \\
\hline Number of pregnancy & $1.47 \pm 0.35$ & $2.59 \pm 0.82$ \\
\hline Number of miscarriage & $0.77 \pm 0.35$ & $2.67 \pm 0.55^{\star \star}$ \\
\hline Number of live birth & $1.56 \pm 0.35$ & $0.00 \pm 0.00^{\star \star}$ \\
\hline
\end{tabular}

RSA, recurrent spontaneous abortion; BMI, body mass index.

${ }^{* *} p<0.01$

significantly lower number of live births than the control group $(p<0.01)$.

\section{Expression of CXCL5 Is Downregulated in Villous Tissues of Recurrent Spontaneous Abortion Patients}

To investigate the role of CXCL5 in RSA, we firstly evaluated the expression levels of CXCL5 in placental villous tissues from 15 RSA patients and 13 normal controls by RT-PCR. Lower expression of CXCL5 was found in the RSA group than in the control group (Figure 1A). In addition, to further identify immunolocalization and compare CXCL5 levels, human placental villous tissue sections were stained via immunohistochemistry (IHC) assay. A weaker stain of CXCL5 was found in villous tissues from RSA patients (Figures 1B, C). These data together suggested that CXCL5 expression was downregulated in villous tissues of RSA patients and that lower levels of CXCL5 were positively associated with RSA.

\section{Expression of CXCR2 in Trophoblast of Human Placental Villous Tissue}

CK7 was recommended as an identification marker for trophoblast (31). To confirm the CXCR2 expression in the trophoblast of human placental villous, we performed colocalization of CXCR2 and CK7 in human placental villous
A

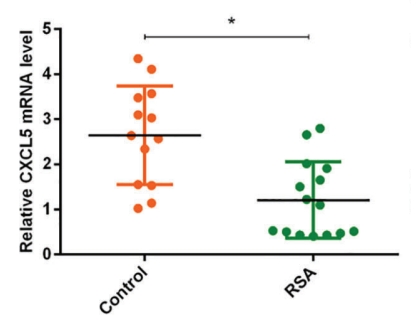

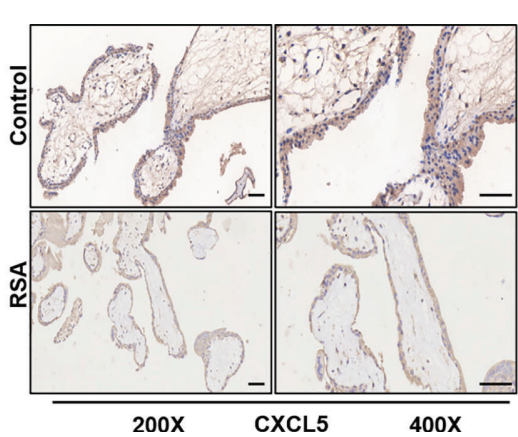

C

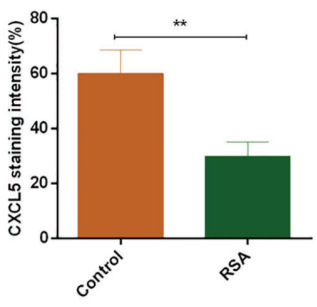

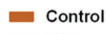

RSA

FIGURE 1 | CXCL5 expression in human placental villous tissue from the RSA group and the control group. (A) Expression of CXCL5 mRNA in the RSA group ( $\mathrm{n}=15$ and control group $(n=13)$ by RT-PCR. (B, C) Representative immunohistochemical staining images and quantification of CXCL5 in the RSA group ( $\mathrm{n}=15)$ and normal group $(n=13)$. Relative gene expression was normalized to GAPDH. ImageJ is used to quantify the staining intensity. Magnification, $\times 200$ and $\times 400$. Scale bars $=100$ $\mu \mathrm{m}$. Data are presented as the mean $\pm \mathrm{SD}$. Results are reported as fold change compared with the control group. ( $\left.{ }^{\star} p<0.05,{ }^{\star \star} p<0.01\right)$. RSA, recurrent spontaneous abortion; mRNA, messenger RNA; RT-PCR, quantitative real-time polymerase chain reaction; CXCL5, C-X-C motif chemokine ligand 5; IHC, immunohistochemistry. 
tissue with the immunofluorescence assay. The results confirmed that CXCR2 was expressed in the CK7-labeled trophoblast and suggested that CXCL5 can bind to the trophoblast cells to exert its functions (Figures 2A-D).

\section{CXCL5 Promotes Trophoblast Migration and Invasion via Inducing the Epithelial-to- Mesenchymal Transition Process}

To investigate the effects of CXCL5 on the migration and invasion of trophoblast, we conducted Transwell and scratch wound healing experiments using HTR-8 cells. The results showed that rhCXCL5-stimulated trophoblast cells performed quicker migration than did the control group (Figures 3A, B). Similarly, CXCL5-treated groups also showed an increased cell invasion potential than did the control group (Figures 3C, D). These results demonstrated that CXCL5 can significantly enhance trophoblast migration and invasion in vitro. EMT was regarded as an important process via which trophoblast acquired invasive ability (32). To gain insight into whether CXCL5 promoted trophoblast migration and invasion via inducing the EMT process, we detected the expression of EMT markers. Western blotting results revealed that CXCL5 treatment significantly decreased the expression of the epithelial marker E-cadherin and increased the expression of the mesenchymal markers $\mathrm{N}$-cadherin and vimentin in HTR-8 cells (Figures 3E, F).
Based on these findings, CXCL5 can promote trophoblast migration and invasion via inducing the EMT process.

\section{CXCL5 Activates PI3K/AKT/ERK1/2 Pathway to Induce the Epithelial-to- Mesenchymal Transition Process}

Studies reported that CXCL5 induced the EMT process in cancer cells by activating ERK/Elk-1/Snail, AKT/GSK3 $\beta / \beta$-catenin, or ERK/Snail signaling pathways $(33,34)$. To explore the potential signaling pathway that CXCL5 induced EMT in trophoblast cells, we detected the activity of these pathways. Western blotting analysis showed that the levels of phosphorylation AKT remarkably increased in CXCL5-treated HTR-8 cells, while the total AKT levels did not change (Figure 4A). Consistently, PI3K levels also increased. In addition, we also observed apparent activation of p-ERK1/2 (Figure 4A). Next, we selected inhibitors specific to ERK (PD98059) and PI3K/AKT (LY294002) pathways for further exploration. A notable blocking effect of LY294002 and PD98059 was observed on p-AKT and p-ERK1/2 levels, respectively (Figure 4B). Remarkably, p-ERK1/2 levels also decreased when the PI3K/AKT was inhibited with LY294002, which suggested that ERK1/2 may act as a direct downstream effector of PI3K/AKT signaling (Figure 4B). Next, inhibitor pretreatment was initiated $2 \mathrm{~h}$ before CXCL5 treatment in HTR-8 cells. The results showed that PD98059 pretreatment
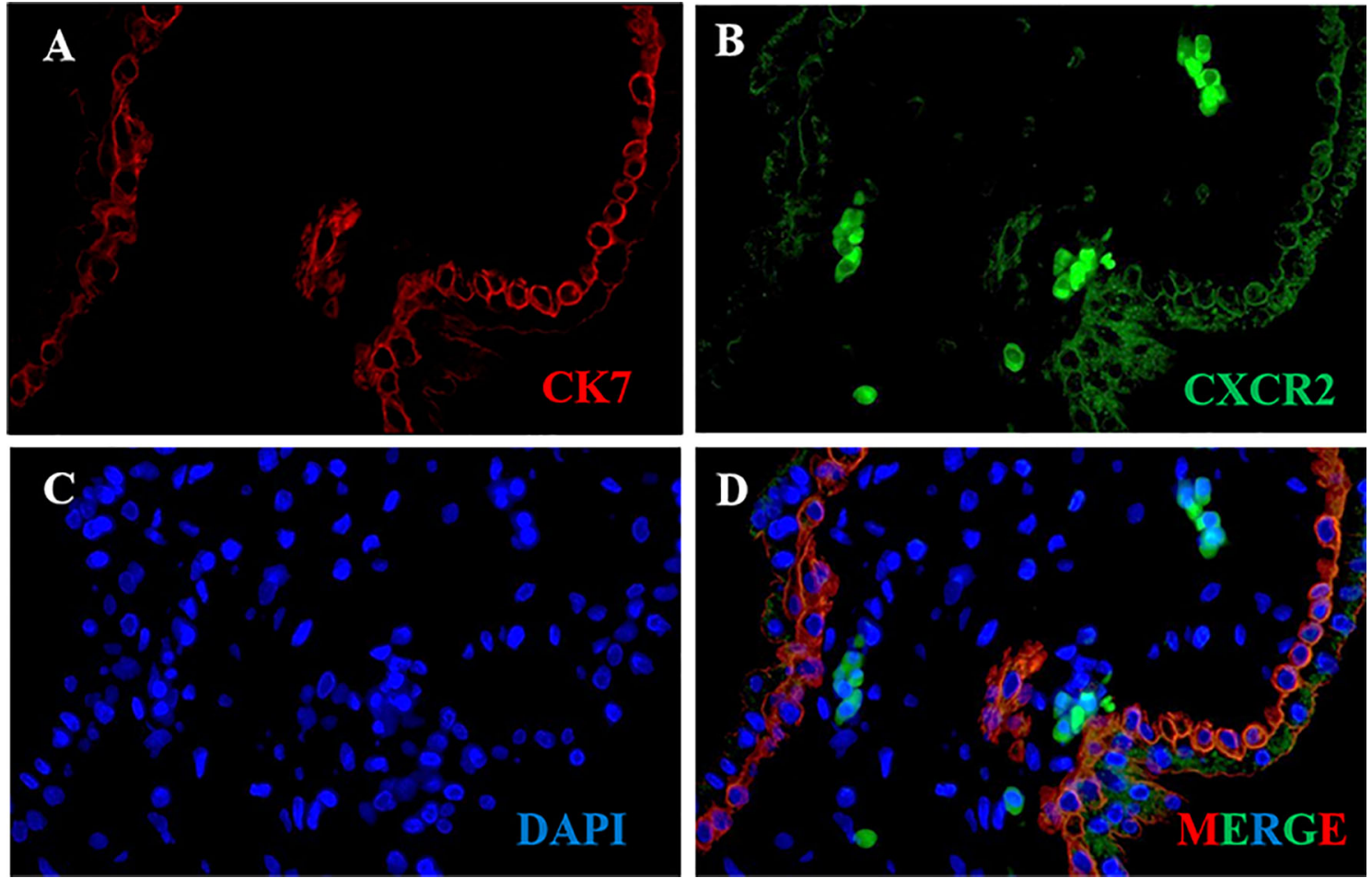

FIGURE 2 | CXCR2 expression in trophoblast of human placental villous tissues. Paraffin-embedded tissue sections were double-stained for immunofluorescence. (A) The CK7-labeled trophoblast cells are indicated by red signals. (B) The CXCR2 protein is indicated by green signals. (C) The DAPI-stained nuclei are indicated by blue signals. (D) CXCR2 was expressed in CK7-labeled trophoblast cells and strongly indicated the colocalization of CK7 and CXCR2. CK7, cytokeratin 7; CXCR2, C-X-C motif chemokine receptor 2; DAPI, 4'-6-diamidino-2-phenylindole. 


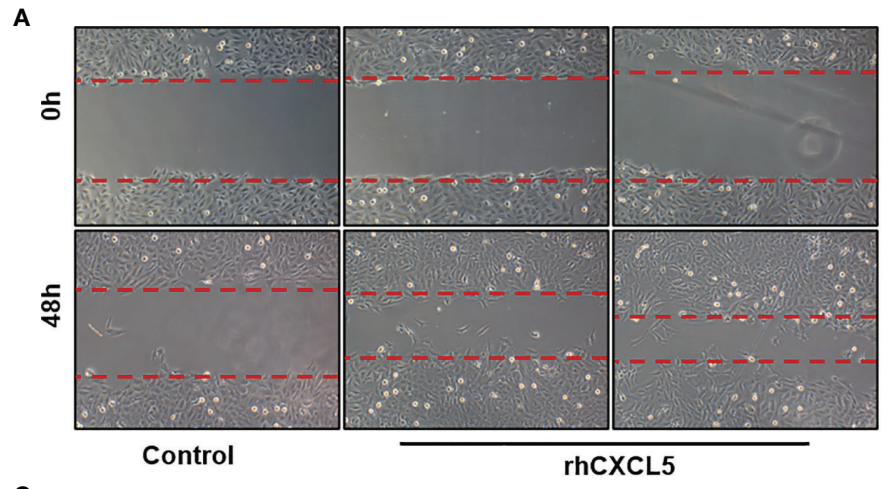

c

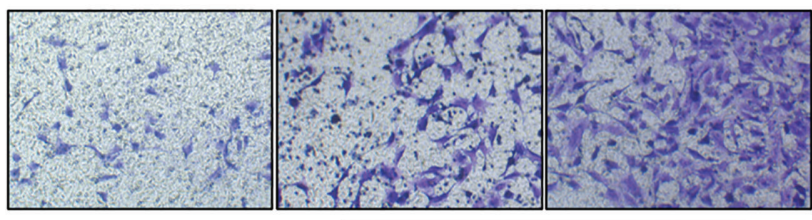

Control

E

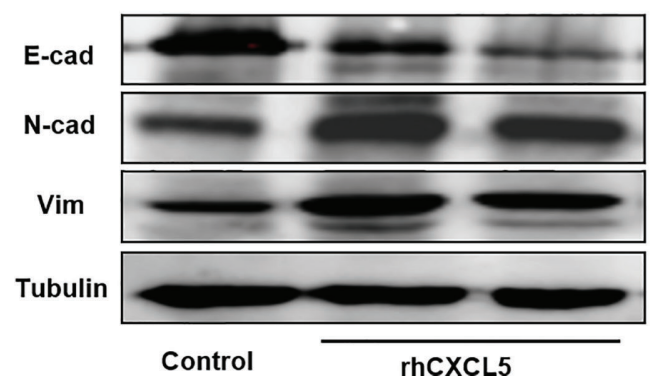

B

D
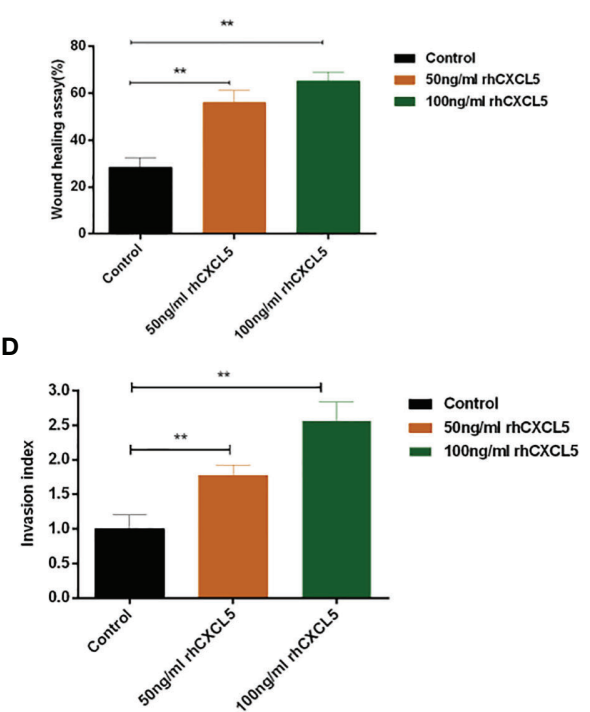

$\mathbf{F}$

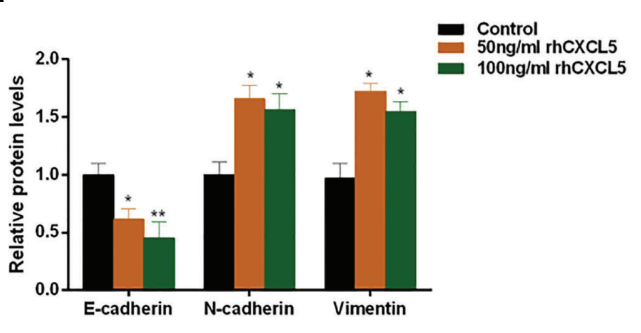

FIGURE 3 | CXCL5 induces the EMT process to promote trophoblast migration and invasion. HTR-8 cells were treated with 0, 50, and 100 $\mathrm{ng} / \mathrm{ml}$ of rhCXCL5 for 48 h. (A, B) Scratch wound healing assay and quantitation were performed to examine trophoblast migration ability. (C, D) Matrigel invasion assay and quantitation were used to detect trophoblast invasiveness ability. (E, F) Western blotting and quantitation were determined to examine the expression levels of E-cadherin, $\mathrm{N}$ cadherin, and vimentin. Representative images of migratory and invasive cells are presented. Data are presented as the mean \pm SD. Results are reported as fold change compared with the control group $\left({ }^{*} p<0.05,{ }^{* *} p<0.01\right)$. rhCXCL5, recombinant CXCL5; EMT, epithelial-to-mesenchymal transition; E-cad, E-cadherin; Ncad, N-cadherin; Vim, vimentin.

significantly reduced the expression of $\mathrm{N}$-cadherin and vimentin but increased the expression of E-cadherin compared with CXCL5 treated alone in trophoblast cells (Figure 4C), which indicated that PD98059 reversed the CXCL5-induce EMT process. In addition, wound healing assay and Matrigel invasion assay results also showed that the invasive and migratory activities of cells were reduced when the ERK1/2 pathway was inhibited (Figures 4E, F). Similar results were observed in the LY294002 treatment group (Figures 4D, G-H). Taken together, these results confirmed that CXCL5 activated PI3K/AKT/ERK1/2 pathway to induce the EMT process of trophoblast cells. We also checked the E-cadherin and $\mathrm{N}$ cadherin expression in human placental villous tissue specimens with IHC assay. The results showed that E-cadherin expression was upregulated (Figures 5A, B) and $\mathrm{N}$-cadherin expression was downregulated (Figures 5C, D) in placental villous tissues from RSA patients compared with the controls.

\section{Estrogen, Progesterone, Human Chorionic Gonadotropin, and Decidual Stromal Cells Regulate CXCL5/CXCR2 Expression of Trophoblast}

Extensive evidence revealed that reproductive hormones and DSCs directly or indirectly affected chemokines expression (3537). Therefore, to confirm their impacts on CXCL5 and CXCR2 expression of trophoblast cells, we cultured HTR-8 cells in the presence or absence of E, P, or HCG. The results are displayed in Figures 6A, B. We found that HCG increased CXCR2 expression $(p<0.01)$ but did not affect CXCL5 expression in HTR-8 cells $(p>0.05)$. E was observed to downregulate CXCL5 levels $(p<0.01)$ but did not affect CXCR2 expression $(p>0.05)$. P did not affect CXCR2 $(p>0.05)$ but increased CXCL5 mRNA expression $(p<0.05)$. To assess the effect of DSCs on CXCL5 and CXCR2 of trophoblast cells, we used a co-culture model of different ratios of HTR-8 cells and DSCs, as shown in Figure 6C. 
A

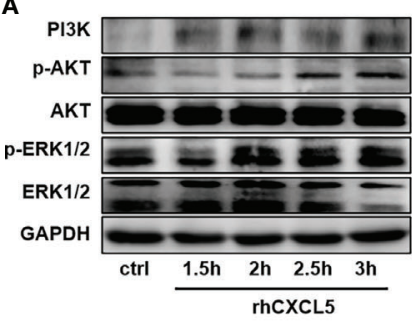

C

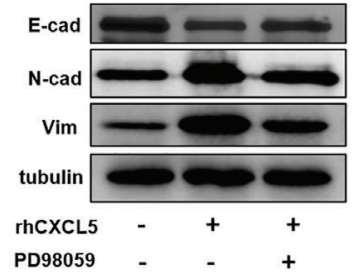

B

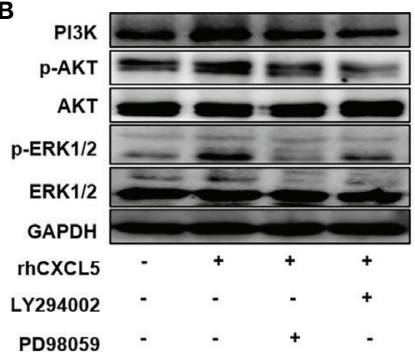

D

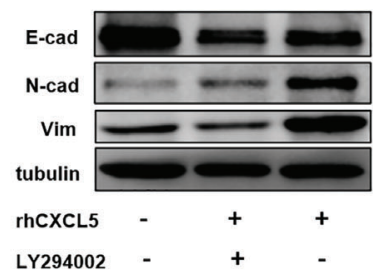

E
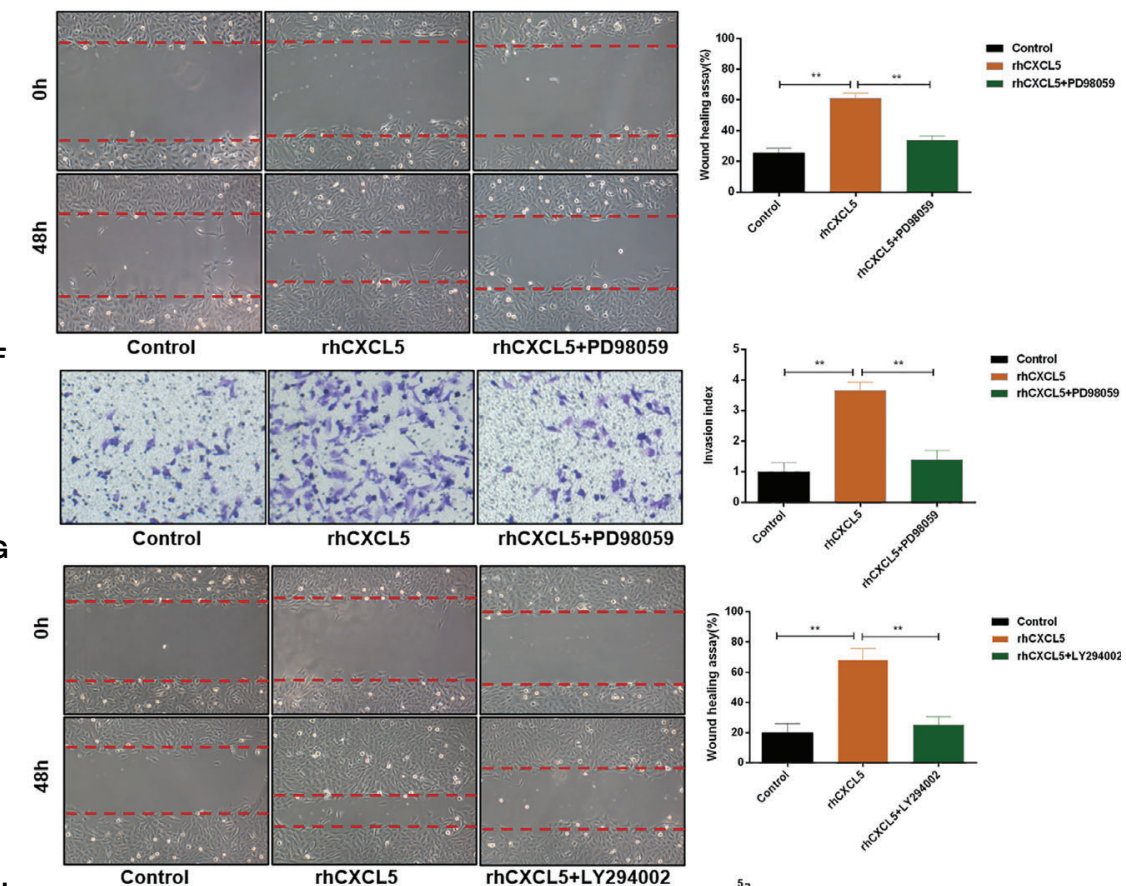

- Control

mCXCLS+P098059

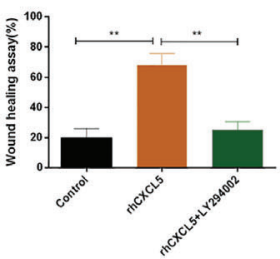

E control

thCXCL5+L $129400:$
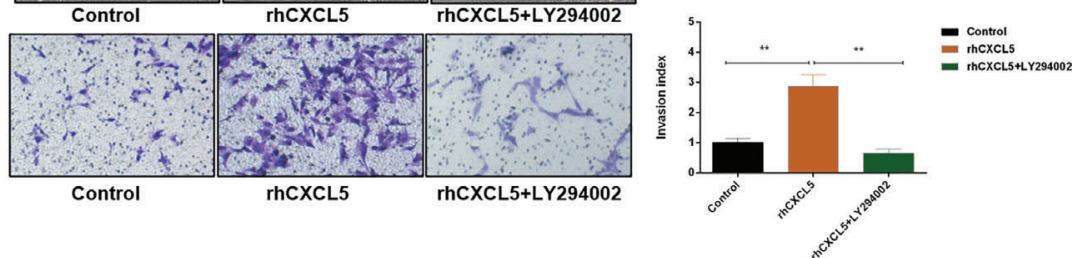

FIGURE 4 | CXCL5 activates PI3K/AKT/ERK1/2 signaling pathway to induce the EMT process of trophoblast cells. HTR-8 cells were pretreated with LY294002 (a PI3K/AKT inhibitor) or PD98059 (an ERK1/2 inhibitor) before treatment with rhCXCL5. (A) Western blotting analysis showed the activation of PI3K/AKT and ERK1/2 pathways in HTR-8 cells stimulated with rhCXCL5 for 1.5, 2, 2.5, and $3 \mathrm{~h}$. (B) Western blotting analysis presented a notable blocking effect of LY294002 and PD98059 on p-AKT or p-ERK1/2, respectively. (C, D) Western blotting was performed to examine the expression of E-cadherin, N-cadherin, and vimentin in HTR-8 cells stimulated with PD98059 or LY294002 for 48 h. (E, F) Scratch wound healing and Matrigel invasion assays were performed to detect trophoblast migration and invasion abilities after stimulation with PD98059 for 48 h. (G, H) Scratch wound healing and Matrigel invasion assays were used to examine trophoblast migration and invasion abilities after stimulation with LY294002 for $48 \mathrm{~h}$. Data are expressed as the mean \pm SD. Column charts were used for quantifications of the migration assay and invasion assays. Results are reported as fold change compared with the control group $\left({ }^{*} p<0.01\right)$. CXCL5, C-X-C motif chemokine ligand 5 . 
A

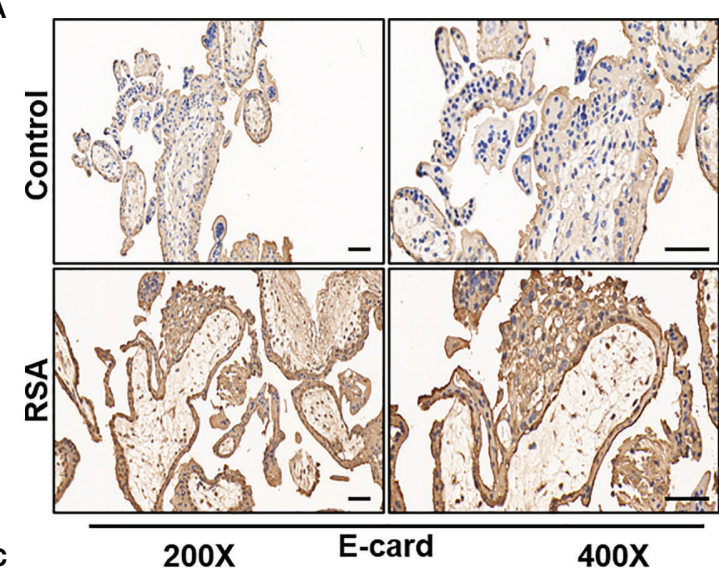

c

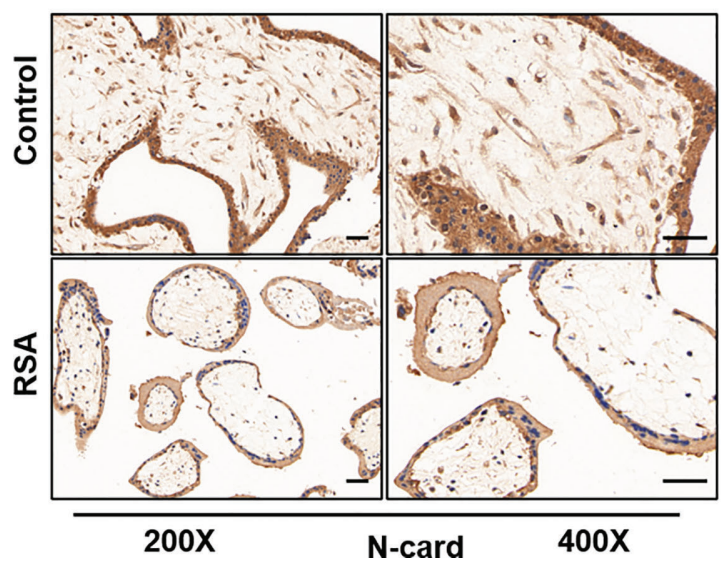

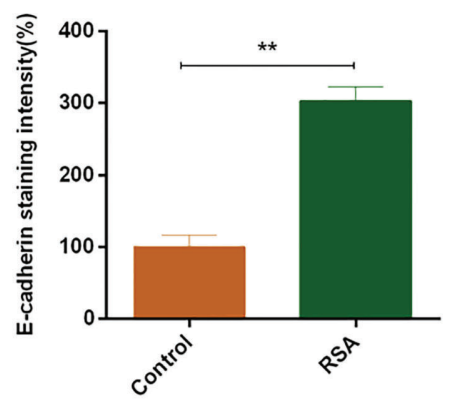

Control

RSA

D

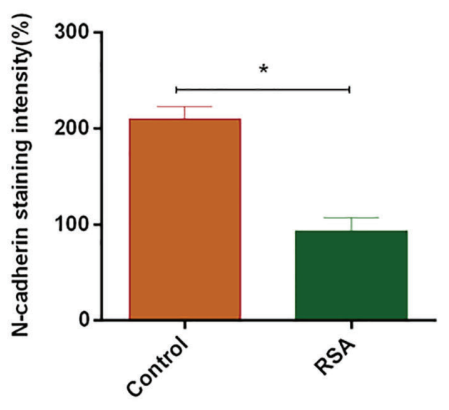

$\square$ Control

- RSA

FIGURE 5 | Expression of E-cadherin and N-cadherin in human placental villous tissues from the RSA group and the control group. Placental villous tissues from women with RSA ( $n=15)$ and normal controls $(n=13)$ were used for the $I H C$ assay. (A, B) Representative immunohistochemical staining images and quantification of E-cadherin in the RSA group $(n=15)$ and normal group $(n=13)$. (C, D) Representative immunohistochemical staining images and quantification of $N$-cadherin in the RSA group $(n=15)$ and normal group $(n=13)$. The staining intensity is quantified using ImageJ. Magnification, $\times 200$ and $\times 400$. Scale bars $=100 \mu \mathrm{m}$. Graphs show the mean $\pm \mathrm{SD}$. Results are reported as fold change compared with the control group $\left({ }^{\star} p<0.05,{ }^{* *} p<0.01\right)$. RSA, recurrent spontaneous abortion; $\mathrm{IHC}$, immunohistochemistry.

The results indicated that DSCs can promote the expression of CXCL5 and CXCR2 of trophoblast cells, even when cultured at a lower ratio (DSCs: HTR-8 cells, 1:4; Figures 6D, E).

\section{DISCUSSION}

RSA is the most common pregnancy-related complication affecting reproductive-age women. Accumulating evidence has demonstrated that trophoblast invasion is closely associated with embryo implantation and placentation and thus plays an important role in the establishment and maintenance of pregnancy. Moreover, studies reported that insufficient invasion of trophoblast cells can lead to the occurrence of RSA $(10,12)$. Our previous work also showed that miR-27a-3p/ USP25 axis participated in the pathogenesis of RSA through inhibiting trophoblast migration and invasion (11). In the current study, we found that CXCL5 levels were downregulated in villous tissues from RSA patients. Furthermore, we demonstrated that CXCL5 induced the EMT process to promote trophoblast invasion and migration through $\mathrm{PI} 3 \mathrm{~K} / \mathrm{AKT} / \mathrm{ERK} 1 / 2$ signaling pathway. These results together demonstrate that the downregulation of CXCL5 in villous tissue plays an essential role in the pathogenesis of RSA.

The maternal-fetal interface exists an abundant chemokines network, which exhibits main functions in inflammation, immune tolerance, and trophoblast invasion during early human pregnancy (14). Substantial studies have reported the relationship between chemokines and trophoblast invasion. Zhang et al. revealed that CXCL6 restricted human trophoblast migration and invasion in vitro (38). Wang et al. pointed out that both exogenous and endogenous CXCL3 regulated trophoblast cells invasion $(27,39)$. Similar results have been identified in CCL24, CXCL16, CXCL14, CCL14, and CCL17 (37, 40-43). Chemokines have been widely reported in the field of cancer and are associated with angiogenesis, invasion, and metastatic 


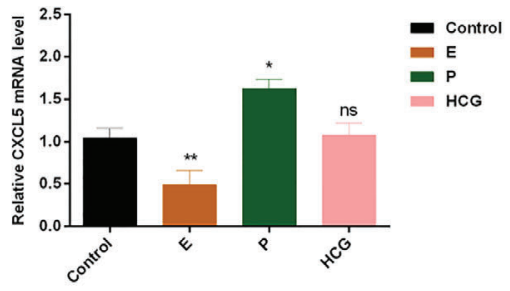

B

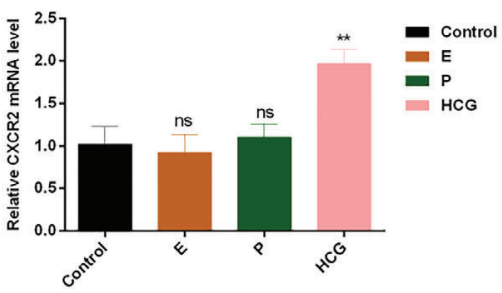

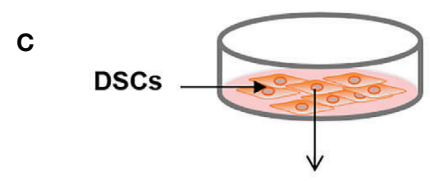
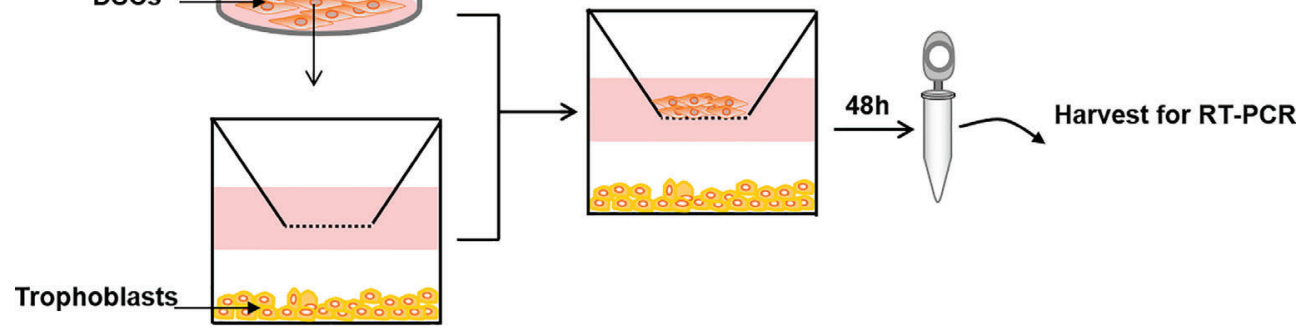

D

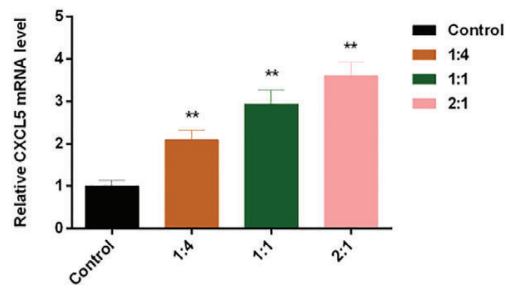

E

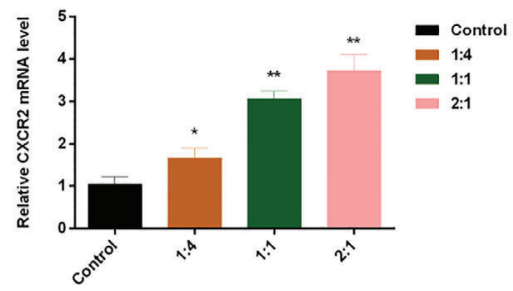

FIGURE 6 | E, P, HCG, and DSCs regulate CXCL5/CXCR2 expression of trophoblast cells. HTR-8 cells were treated with E, P, and HCG at the indicated concentrations for $48 \mathrm{~h}$. (A, B) RT-PCR was used to detect the expression levels of CXCL5 and CXCR2. (C) A co-culture system of HTR-8 cells and DSCs was taken for 48 h. (D, E) RT-PCR was used to investigate the impact of DSCs on CXCL5 and CXCR2 expression of trophoblast cells. Graphs show the mean \pm SD. Results are reported as fold change compared with the control group $\left({ }^{*} p<0.05,{ }^{* *} p<0.01\right.$, ns, no significant difference, $\left.p>0.05\right)$. E, estrogen; $\mathrm{P}$, progesterone; HCG, human chorionic gonadotropin; DSCs, decidual stromal cells; RT-PCR, quantitative real-time PCR.

potential of tumors. For instance, Mao et al. found that CXCL5 can enhance gastric cancer cells migration and invasion ability via inducing the EMT process (34). Kodama et al. reported that the CCL3/CCR5 axis contributed to esophageal squamous cell migration and invasion (44). Trophoblast cells have much in common with tumor cells (24). Therefore, our current study investigated the effect of CXCL5 on trophoblast invasion. As described above, we firstly detected CXCL5 expression in villous samples from RSA patients and control patients and found a significant downregulation of CXCL5 in the former. Next, we confirmed that human villous trophoblast expressed CXCR2, which was consistent with a previous study, and suggested CXCL5 can exert impacts on trophoblast cells via the receptorligand binding mechanism (25). We further conducted in vitro experiments using HTR-8 cells. Exploring trophoblast invasion relies on a suitable trophoblast line because obtaining pure, primary, first-trimester human trophoblast remains a challenge. Compared with BeWo, JEG-3, and JAR, which are highly malignant and have a substantially different transcriptomic profile from EVTs, the HTR-8/SVeo cell line is reported to contain a heterogeneous population of trophoblasts and has been widely used to investigate EVT biology and functions (45, 46). Our results showed that CXCL5 can promote trophoblast cells migration and invasion. Therefore, the downregulation of CXCL5 in villous tissues of RSA patients leads to inadequate trophoblast invasion and the development of RSA.

EMT is firstly described by Elizabeth Hay and is referred to as a multistep dynamic cellular phenomenon in which epithelial cells lose their cell-cell adhesions and gain migratory and invasive traits that are typical of mesenchymal cells (47). This process is characterized by loss of the membranous epithelial marker Ecadherin, increase of mesenchymal markers including vimentin and $\mathrm{N}$-cadherin, and enhanced migratory and invasive behaviors. It has been reported that EMT participates in embryonic development, tissue repair, and cancer metastasis (48-50). We provided the first evidence that CXCL5 induced the EMT process to enhance trophoblast invasion and migration. Additionally, we also observed the reversal of EMT in CXCL5-reduced villous tissues from RSA patients. These data emphasized the importance of EMT in pregnancy. Interestingly, recent studies have also found that EMT 


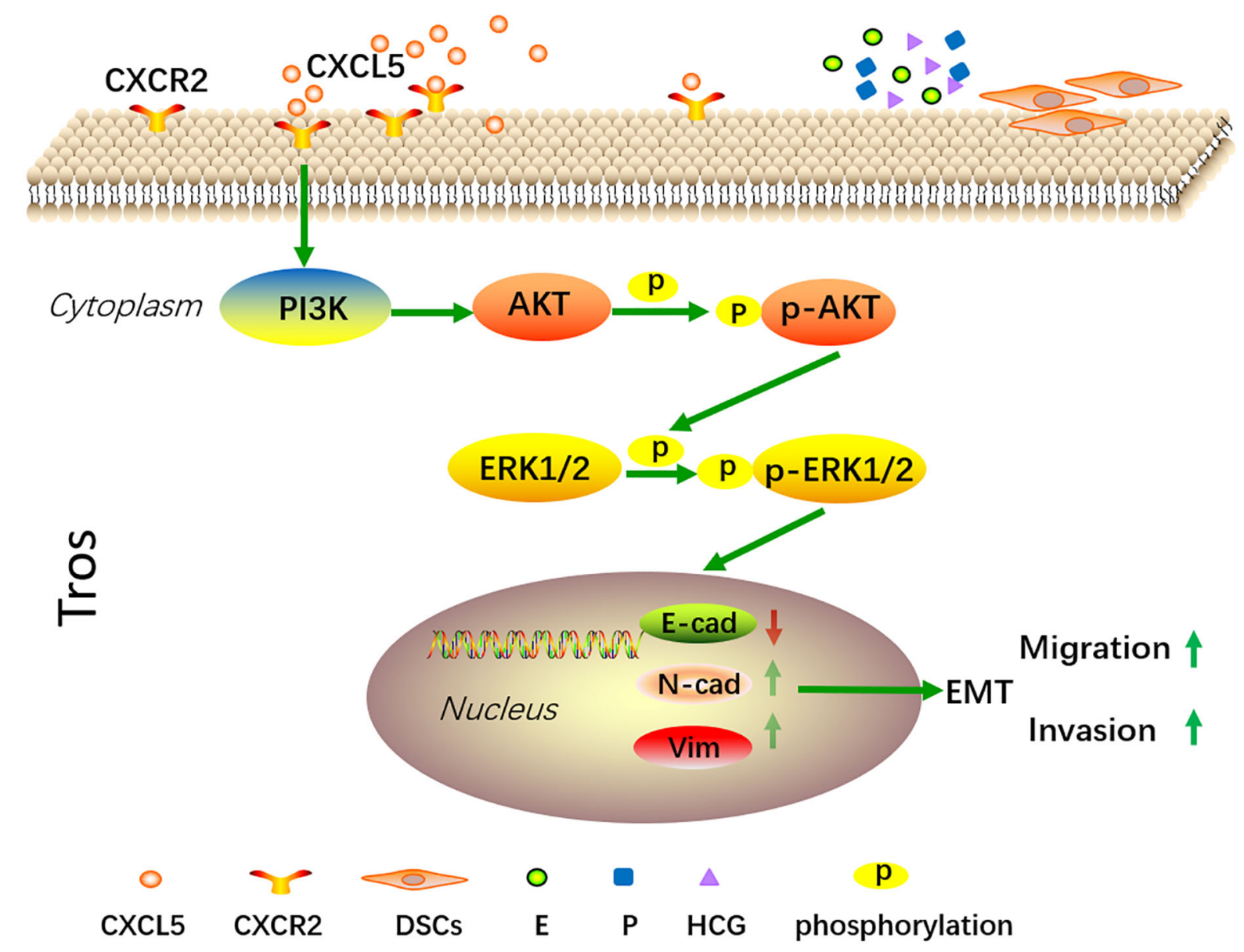

FIGURE 7 | A schematic diagram of CXCL5 promoting trophoblast migration, invasion, and EMT process through PI3K/AKT/ERK1/2 signaling pathway. Trophoblast cells express CXCR2, which is the receptor of CXCL5. EXogenous CXCL5 combined with CXCR2 promotes the EMT process through the activation of the PI3K/AKT/ERK1/2 signaling pathway and consequently increases the invasiveness and migration of trophoblast cells. Conversely, downregulation of CXCL5 results in insufficient invasion and therefore is correlated with the pathology of RSA. In addition, E, P, HCG, and DSCs participate in regulating CXCL5 and CXCR2 expression of trophoblast cells within the microenvironment of pregnancy at the maternal-fetal interface. CXCL5, C-X-C motif chemokine ligand 5; EMT, epithelial-tomesenchymal transition.

has an intimate association with aerobic glycolysis $(51,52)$. In addition, Ma et al. have reported that lactic acid, which is a critical metabolite product of aerobic glycolysis, plays a role in trophoblast invasion and angiogenesis (53). However, whether this effect is mediated by EMT induction to link pregnancy requires further investigation.

PI3K/AKT and ERK pathways are reported to play significant roles in the CXCL5-induced cell invasion and the EMT process. Qiu et al. found that CXCL5/CXCR2 axis contributed to the EMT of nasopharyngeal carcinoma cells by ERK/GSK-3 $\beta /$ snail signaling (23). Zhao et al. reported that tumor-derived CXCL5 promoted human colorectal cancer metastasis through the activation of ERK/Elk-1/Snail and AKT/GSK3 $\beta / \beta$-catenin pathways (33). In our present study, CXCL5 can activate PI3K/ AKT/ERK1/2 pathway to induce the EMT process and enhance trophoblast invasion. These data again highlight the importance of PI3K/AKT and ERK pathway. In addition, PI3K/AKT signaling is also identified as a potential therapeutic target. Epidemiological studies and meta-analyses have shown that the use of statins is closely associated with a reduced incidence of colorectal cancer (54-56). Recently, a new study has revealed that statins can target inhibition PI3K/AKT/mTOR signaling and thus acts on colorectal cancer progression (57). Remarkably, another research has found that pravastatin can successfully prevent fetal death in a pregnant woman with a history of four consecutive pregnancy losses (58). However, whether this effect depends on PI3K/AKT pathway needs more exploration.

It is reported that reproductive hormones E, P, and HCG play important roles during pregnancy and can affect the expression of chemokines within the maternal-fetal microenvironment (36). Currently, we found that $\mathrm{P}$ significantly increased CXCL5 expression while $\mathrm{E}$ had an opposite function. Our results also showed that HCG upregulated CXCR2 expression, although it did not affect CXCL5 expression. These findings indicated that CXCL5/ 
CXCR2 axis is regulated by local hormones. There is also a close dialogue between trophoblast cells and maternal DSCs at the maternal-fetal interface (59). Co-culture systems comprising decidual fragments and trophoblasts have been widely used to explore their relationship. Li et al. reported that DSCs promoted CCR3 levels of trophoblast cells (37). Our current result confirmed that DSCs significantly promoted CXCL5 and CXCR2 expression of trophoblast cells. However, whether DSCs can induce trophoblast cells to secrete CXCL5 and participate in the regulation of trophoblast invasion deserve further research in future work.

In summary, our present data confirm that CXCL5 levels are significantly lower in human villous tissue from RSA patients. We also demonstrate that CXCL5 can promote trophoblast invasion, migration, and EMT process through PI3K/AKT/ ERK1/2 pathway. Taken together, these data indicate that CXCL5 downregulation in villous tissue is correlated with RSA. This provides us with more insights into the molecular pathogenesis of RSA. In addition, we also found that E, P, HCG, and DSCs regulate the expression of CXCL5/CXCR2 in a cell manner. These findings illustrate a new dialogue among chemokines, trophoblast cells, and reproductive hormones in the microenvironment of the maternal-fetal interface (Figure 7).

\section{DATA AVAILABILITY STATEMENT}

The datasets presented in this study can be found in online repositories. The names of the repository/repositories and accession number(s) can be found in the article/supplementary material.

\section{REFERENCES}

1. Definitions of Infertility and Recurrent Pregnancy Loss: A Committee Opinion. Fertil Steril (2020) 113(3):533-5. doi: 10.1016/j.fertnstert. 2019.11.025

2. Pereza N, Ostojić S, Kapović M, Peterlin B. Systematic Review and MetaAnalysis of Genetic Association Studies in Idiopathic Recurrent Spontaneous Abortion. Fertil Steril (2017) 107(1):150-9.e2. doi: 10.1016/ j.fertnstert.2016.10.007

3. Wang SW, Zhong SY, Lou LJ, Hu ZF, Sun HY, Zhu HY. The Effect of Intravenous Immunoglobulin Passive Immunotherapy on Unexplained Recurrent Spontaneous Abortion: A Meta-Analysis. Reprod BioMed Online (2016) 33(6):720-36. doi: 10.1016/j.rbmo.2016.08.025

4. Garrido-Gimenez C, Alijotas-Reig J. Recurrent Miscarriage: Causes, Evaluation and Management. Postgrad Med J (2015) 91(1073):151-62. doi: 10.1136/postgradmedj-2014-132672

5. Staun-Ram E, Shalev E. Human Trophoblast Function During the Implantation Process. Reprod Biol Endocrinol (2005) 3:56. doi: 10.1186/ 1477-7827-3-56

6. Ji L, Brkić J, Liu M, Fu G, Peng C, Wang YL. Placental Trophoblast Cell Differentiation: Physiological Regulation and Pathological Relevance to Preeclampsia. Mol Aspects Med (2013) 34(5):981-1023. doi: 10.1016/ j.mam.2012.12.008

7. Fisher SJ, Damsky CH. Human Cytotrophoblast Invasion. Semin Cell Biol (1993) 4(3):183-8. doi: 10.1006/scel.1993.1022

8. Huppertz B. The Critical Role of Abnormal Trophoblast Development in the Etiology of Preeclampsia. Curr Pharm Biotechnol (2018) 19(10):771-80. doi: 10.2174/1389201019666180427110547

9. Huppertz B. Traditional and New Routes of Trophoblast Invasion and Their Implications for Pregnancy Diseases. Int J Mol Sci (2019) 21(1):289. doi: $10.3390 /$ ijms 21010289

\section{ETHICS STATEMENT}

The studies involving human participants were reviewed and approved by the ethics committee of Renmin Hospital of Wuhan University. The patients/participants provided their written informed consent to participate in this study. Written informed consent was obtained from the individual(s) for the publication of any potentially identifiable images or data included in this article.

\section{AUTHOR CONTRIBUTIONS}

TY and YZ supported the research. SZ and JD designed the experiments. SZ performed the experiments and drafted the first version of the manuscript. JW collected the clinical samples and patients' data. TY, YZ, JD, and JY supervised and revised the manuscript. All authors contributed to the article and approved the submitted version.

\section{FUNDING}

This work was supported by the following grants: National Key Research and Development Program of China (Nos. 2018YFC1004601, 2018YFC1002804), the National Natural Science Foundation of China (Nos. 81801540, 81771662, 82101749), and the Fundamental Research Funds for the Central Universities (2042021kf0082).

10. Tian FJ, Qin CM, Li XC, Wu F, Liu XR, Xu WM, et al. Decreased Stathmin-1 Expression Inhibits Trophoblast Proliferation and Invasion and Is Associated With Recurrent Miscarriage. Am J Pathol (2015) 185(10):2709-21. doi: 10.1016/j.ajpath.2015.06.010

11. Ding J, Cheng Y, Zhang Y, Liao S, Yin T, Yang J. The miR-27a-3p/USP25 Axis Participates in the Pathogenesis of Recurrent Miscarriage by Inhibiting Trophoblast Migration and Invasion. J Cell Physiol (2019) 234(11):1995163. doi: $10.1002 /$ jcp. 28593

12. Lv S, Wang N, Lv H, Yang J, Liu J, Li WP, et al. The Attenuation of Trophoblast Invasion Caused by the Downregulation of EZH2 Is Involved in the Pathogenesis of Human Recurrent Miscarriage. Mol Ther Nucleic Acids (2019) 14:377-87. doi: 10.1016/j.omtn.2018.12.011

13. Zlotnik A, Yoshie O, Nomiyama H. The Chemokine and Chemokine Receptor Superfamilies and Their Molecular Evolution. Genome Biol (2006) 7(12):243. doi: $10.1186 / \mathrm{gb}-2006-7-12-243$

14. Du MR, Wang SC, Li DJ. The Integrative Roles of Chemokines at the Maternal-Fetal Interface in Early Pregnancy. Cell Mol Immunol (2014) 11 (5):438-48. doi: $10.1038 / \mathrm{cmi} .2014 .68$

15. Warner JA, Zwezdaryk KJ, Day B, Sullivan DE, Pridjian G, Morris CA. Human Cytomegalovirus Infection Inhibits CXCL12- Mediated Migration and Invasion of Human Extravillous Cytotrophoblasts. Virol J (2012) 9:255. doi: $10.1186 / 1743-422 x-9-255$

16. Chang MS, McNinch J, Basu R, Simonet S. Cloning and Characterization of the Human Neutrophil-Activating Peptide (ENA-78) Gene. J Biol Chem (1994) 269(41):25277-82.

17. Vandercappellen J, Van Damme J, Struyf S. The Role of CXC Chemokines and Their Receptors in Cancer. Cancer Lett (2008) 267(2):226-44. doi: 10.1016/ j.canlet.2008.04.050

18. Keelan JA, Yang J, Romero RJ, Chaiworapongsa T, Marvin KW, Sato TA, et al. Epithelial Cell-Derived Neutrophil-Activating Peptide-78 is Present in Fetal Membranes and Amniotic Fluid at Increased Concentrations With Intra- 
Amniotic Infection and Preterm Delivery. Biol Reprod (2004) 70(1):253-9. doi: 10.1095/biolreprod.103.016204

19. Sepuru KM, Poluri KM, Rajarathnam K. Solution Structure of CXCL5-A Novel Chemokine and Adipokine Implicated in Inflammation and Obesity. PLoS One (2014) 9(4):e93228. doi: 10.1371/journal.pone.0093228

20. Chavey C, Fajas L. CXCL5 Drives Obesity to Diabetes, and Further. Aging (Albany NY) (2009) 1(7):674-7. doi: 10.18632/aging.100064

21. Zhang W, Wang H, Sun M, Deng X, Wu X, Ma Y, et al. CXCL5/CXCR2 Axis in Tumor Microenvironment as Potential Diagnostic Biomarker and Therapeutic Target. Cancer Commun (Lond) (2020) 40(2-3):69-80. doi: $10.1002 / \mathrm{cac} 2.12010$

22. Zhou SL, Zhou ZJ, Hu ZQ, Li X, Huang XW, Wang Z, et al. CXCR2/CXCL5 Axis Contributes to Epithelial-Mesenchymal Transition of HCC Cells Through Activating PI3K/Akt/GSK-3ß/Snail Signaling. Cancer Lett (2015) 358(2):124-35. doi: 10.1016/j.canlet.2014.11.044

23. Qiu WZ, Zhang HB, Xia WX, Ke LR, Yang J, Yu YH, et al. The CXCL5/ CXCR2 Axis Contributes to the Epithelial-Mesenchymal Transition of Nasopharyngeal Carcinoma Cells by Activating ERK/GSK-3ß/Snail Signalling. J Exp Clin Cancer Res (2018) 37(1):85. doi: 10.1186/s13046-0180722-6

24. Ferretti C, Bruni L, Dangles-Marie V, Pecking AP, Bellet D. Molecular Circuits Shared by Placental and Cancer Cells, and Their Implications in the Proliferative, Invasive and Migratory Capacities of Trophoblasts. Hum Reprod Update (2007) 13(2):121-41. doi: 10.1093/humupd/dml048

25. Wu D, Hong H, Huang X, Huang L, He Z, Fang Q, et al. CXCR2 is Decreased in Preeclamptic Placentas and Promotes Human Trophoblast Invasion Through the Akt Signaling Pathway. Placenta (2016) 43:17-25. doi: 10.1016/j.placenta.2016.04.016

26. Jovanović M, Stefanoska I, Radojcić L, Vićovac L. Interleukin-8 (CXCL8) Stimulates Trophoblast Cell Migration and Invasion by Increasing Levels of Matrix Metalloproteinase (MMP)2 and MMP9 and Integrins Alpha5 and Beta1. Reproduction (2010) 139(4):789-98. doi: 10.1530/rep-09-0341

27. Gui S, Ni S, Jia J, Gong Y, Gao L, Zhang L, et al. Inconformity of CXCL3 Plasma Level and Placenta Expression in Preeclampsia and Its Effect on Trophoblast Viability and Invasion. PLoS One (2014) 9(12):e114408. doi: 10.1371/journal.pone. 0114408

28. Whitcomb BW, Schisterman EF, Klebanoff MA, Baumgarten M, Rhoton-Vlasak A, Luo X, et al. Circulating Chemokine Levels and Miscarriage. Am J Epidemiol (2007) 166(3):323-31. doi: 10.1093/aje/ kwm084

29. Fernández-Shaw S, Shorter SC, Naish CE, Barlow DH, Starkey PM. Isolation and Purification of Human Endometrial Stromal and Glandular Cells Using Immunomagnetic Microspheres. Hum Reprod (1992) 7(2):156-61. doi: 10.1093/oxfordjournals.humrep.a137609

30. Irwin JC, de las Fuentes L, Giudice LC. Growth Factors and Decidualization In Vitro. Ann NY Acad Sci (1994) 734:7-18. doi: 10.1111/j.17496632.1994.tb21730.x

31. Moser G, Orendi K, Gauster M, Siwetz M, Helige C, Huppertz B. The Art of Identification of Extravillous Trophoblast. Placenta (2011) 32(2):197-9. doi: 10.1016/j.placenta.2010.11.008

32. Ví́ovac L, Aplin JD. Epithelial-Mesenchymal Transition During Trophoblast Differentiation. Acta Anat (Basel) (1996) 156(3):202-16. doi: 10.1159/ 000147847

33. Zhao J, Ou B, Han D, Wang P, Zong Y, Zhu C, et al. Tumor-Derived CXCL5 Promotes Human Colorectal Cancer Metastasis Through Activation of the ERK/Elk-1/Snail and AKT/GSK3// $\beta$-Catenin Pathways. Mol Cancer (2017) 16(1):70. doi: 10.1186/s12943-017-0629-4

34. Mao Z, Zhang J, Shi Y, Li W, Shi H, Ji R, et al. CXCL5 Promotes Gastric Cancer Metastasis by Inducing Epithelial-Mesenchymal Transition and Activating Neutrophils. Oncogenesis (2020) 9(7):63. doi: 10.1038/s41389020-00249-z

35. Dominguez F, Galan A, Martin JJ, Remohi J, Pellicer A, Simón C. Hormonal and Embryonic Regulation of Chemokine Receptors CXCR1, CXCR4, CCR5 and CCR2B in the Human Endometrium and the Human Blastocyst. Mol Hum Reprod (2003) 9(4):189-98. doi: 10.1093/molehr/gag024

36. Park DW, Yang KM. Hormonal Regulation of Uterine Chemokines and Immune Cells. Clin Exp Reprod Med (2011) 38(4):179-85. doi: 10.5653/ cerm.2011.38.4.179
37. Li H, Meng Y, Shang W, Liu L, Chen X, Yuan M, et al. Chemokine CCL24 Promotes the Growth and Invasiveness of Trophoblasts Through ERK1/2 and PI3K Signaling Pathways in Human Early Pregnancy. Reproduction (Cambridge Engl) (2015) 150(5):417-27. doi: 10.1530/rep-15-0119

38. Zhang H, Hou L, Li CM, Zhang WY. The Chemokine CXCL6 Restricts Human Trophoblast Cell Migration and Invasion by Suppressing MMP-2 Activity in the First Trimester. Hum Reprod (2013) 28(9):2350-62. doi: 10.1093/humrep/det258

39. Wang H, Wang T, Dai L, Cao W, Ye L, Gao L, et al. Effects of CXCL3 on Migration, Invasion, Proliferation and Tube Formation of Trophoblast Cells. Placenta (2018) 66:47-56. doi: 10.1016/j.placenta.2018.05.004

40. Huang Y, Zhu XY, Du MR, Wu X, Wang MY, Li DJ. Chemokine CXCL16, a Scavenger Receptor, Induces Proliferation and Invasion of First-Trimester Human Trophoblast Cells in an Autocrine Manner. Hum Reprod (2006) 21 (4):1083-91. doi: 10.1093/humrep/dei436

41. Kuang H, Chen Q, Zhang Y, Zhang L, Peng H, Ning L, et al. The Cytokine Gene CXCL14 Restricts Human Trophoblast Cell Invasion by Suppressing Gelatinase Activity. Endocrinology (2009) 150(12):5596-605. doi: 10.1210/ en.2009-0570

42. Hannan NJ, Jones RL, White CA, Salamonsen LA. The Chemokines, CX3CL1, CCL14, and CCL4, Promote Human Trophoblast Migration at the FetoMaternal Interface. Biol Reprod (2006) 74(5):896-904. doi: 10.1095/ biolreprod.105.045518

43. Li CM, Hou L, Zhang H, Zhang WY. CCL17 Induces Trophoblast Migration and Invasion by Regulating Matrix Metalloproteinase and Integrin Expression in Human First-Trimester Placenta. Reprod Sci (2014). doi: 10.1177/ 1933719113519170

44. Kodama T, Koma YI, Arai N, Kido A, Urakawa N, Nishio M, et al. CCL3CCR5 Axis Contributes to Progression of Esophageal Squamous Cell Carcinoma by Promoting Cell Migration and Invasion via Akt and ERK Pathways. Lab Invest (2020) 100(9):1140-57. doi: 10.1038/s41374-020-0441-4

45. Abbas Y, Turco MY, Burton GJ, Moffett A. Investigation of Human Trophoblast Invasion In Vitro. Hum Reprod Update (2020) 26(4):501-13. doi: 10.1093/humupd/dmaa017

46. Msheik H, Azar J, El Sabeh M, Abou-Kheir W, Daoud G. HTR-8/SVneo: A Model for Epithelial to Mesenchymal Transition in the Human Placenta. Placenta (2020) 90:90-7. doi: 10.1016/j.placenta.2019.12.013

47. Hay ED. An Overview of Epithelio-Mesenchymal Transformation. Acta Anat (Basel) (1995) 154(1):8-20. doi: 10.1159/000147748

48. Kalluri R, Weinberg RA. The Basics of Epithelial-Mesenchymal Transition. J Clin Invest (2009) 119(6):1420-8. doi: 10.1172/jci39104

49. Acloque H, Adams MS, Fishwick K, Bronner-Fraser M, Nieto MA. EpithelialMesenchymal Transitions: The Importance of Changing Cell State in Development and Disease. J Clin Invest (2009) 119(6):1438-49. doi: $10.1172 /$ jci38019

50. Heerboth S, Housman G, Leary M, Longacre M, Byler S, Lapinska K, et al. EMT and Tumor Metastasis. Clin Transl Med (2015) 4:6. doi: 10.1186/s40169-015-0048-3

51. Sciacovelli M, Frezza C. Metabolic Reprogramming and Epithelial-toMesenchymal Transition in Cancer. FEBS J (2017) 284(19):3132-44 doi: 10.1111/febs.14090

52. Yu J, Li J, Chen Y, Cao W, Lu Y, Yang J, et al. Snail Enhances Glycolysis in the Epithelial-Mesenchymal Transition Process by Targeting FBP1 in Gastric Cancer. Cell Physiol Biochem (2017) 43(1):31-8. doi: 10.1159/000480314

53. Ma LN, Huang XB, Muyayalo KP, Mor G, Liao AH. Lactic Acid: A Novel Signaling Molecule in Early Pregnancy? Front Immunol (2020) 11:279. doi: $10.3389 /$ fimmu.2020.00279

54. Cardwell CR, Hicks BM, Hughes C, Murray LJ. Statin Use After Colorectal Cancer Diagnosis and Survival: A Population-Based Cohort Study. J Clin Oncol (2014) 32(28):3177-83. doi: 10.1200/jco.2013.54.4569

55. Hillmer M, Juurlink DN. Do Statins Decrease the Risk of Colorectal Cancer? Cmaj (2005) 173(7):753. doi: 10.1503/cmaj.051076

56. Li Y, He X, Ding Y, Chen H, Sun L. Statin Uses and Mortality in Colorectal Cancer Patients: An Updated Systematic Review and Meta-Analysis. Cancer Med (2019) 8(6):3305-13. doi: 10.1002/cam4.2151

57. Ouahoud S, Jacobs RJ, Peppelenbosch MP, Fühler GM, Heijmans J, Diks S, et al. Correction: Kinome-Wide Analysis of the Effect of Statins in Colorectal Cancer. Br J Cancer (2021) 125(1):144. doi: 10.1038/s41416021-01426-6 
58. Chaiworapongsa T, Romero R, Korzeniewski SJ, Chaemsaithong P, Hernandez-Andrade E, Segars JH, et al. Pravastatin to Prevent Recurrent Fetal Death in Massive Perivillous Fibrin Deposition of the Placenta (MPFD). J Matern Fetal Neonatal Med (2016) 29(6):855-62. doi: 10.3109/ 14767058.2015.1022864

59. Sharma S, Godbole G, Modi D. Decidual Control of Trophoblast Invasion. Am J Reprod Immunol (2016) 75(3):341-50. doi: 10.1111/aji.12466

Conflict of Interest: The authors declare that the research was conducted in the absence of any commercial or financial relationships that could be construed as a potential conflict of interest.
Publisher's Note: All claims expressed in this article are solely those of the authors and do not necessarily represent those of their affiliated organizations, or those of the publisher, the editors and the reviewers. Any product that may be evaluated in this article, or claim that may be made by its manufacturer, is not guaranteed or endorsed by the publisher.

Copyright $\odot 2021$ Zhang, Ding, Wang, Yin, Zhang and Yang. This is an open-access article distributed under the terms of the Creative Commons Attribution License (CC BY). The use, distribution or reproduction in other forums is permitted, provided the original author(s) and the copyright owner(s) are credited and that the original publication in this journal is cited, in accordance with accepted academic practice. No use, distribution or reproduction is permitted which does not comply with these terms. 